\title{
Astrocytic Plasticity and Patterned Oxytocin Neuronal Activity: Dynamic Interactions
}

\author{
Yu-Feng Wang and Glenn I. Hatton \\ Department of Cell Biology and Neuroscience, University of California, Riverside, California 92521
}

Astroglial-neuronal interactions are important in brain functions. However, roles of glial fibrillary acidic protein (GFAP) in this interaction remain unclear in acute physiological processes. We explored this issue using the supraoptic nucleus (SON) in lactating rats. At first, we identified the essential role of astrocytes in the milk-ejection reflex (MER) by disabling astrocytic functions via intracerebroventricular application of $\mathrm{L}$-aminoadipic acid (L-AAA). L-AAA blocked the MER and reduced GFAP levels in the SON. In brain slices, L-AAA suppressed oxytocin (OT) neuronal activity and EPSCs. Suckling reduced GFAP in immunocytochemical images and in Western blots, reductions that were partially reversed after the MER. OT, the dominant hormone mediating the MER, reduced GFAP expression in brain slices. Tetanus toxin suppressed EPSCs but did not influence OT-reduced GFAP. Protease inhibitors did not influence OT-reduced GFAP images but blocked the degradation of GFAP molecules. In the presence of OT, transient $12 \mathrm{~mm} \mathrm{~K}{ }^{+}$exposure, simulating effects of synchronized bursts before the MER, reversed OT-reduced GFAP expression. Consistently, suckling first reduced and then increased the expression of aquaporin 4, astrocytic water channels coupled to $\mathrm{K}^{+}$channels. Moreover, GFAP molecules were associated with astrocytic proteins, including aquaporin 4 , actin, and glutamine synthetase and serine racemase. GFAP-aquaporin 4 association decreased during initial suckling and increased after the MER, whereas opposite changes occurred between GFAP and actin. MER also decreased the association between GFAP and glutamine synthetase. These results indicate that suckling elicits dynamic glial neuronal interactions in the SON; GFAP plasticity dynamically reflects OT neuronal activity.

Key words: excitability; Gfa (glial fibrillary acidic protein); imaging; lactation; protein; supraoptic

\section{Introduction}

Astroglial-neuronal interactions are important in brain functions, including homeostatic, metabolic, signaling, trophic, and pathophysiological regulation (Tekkok and Ransom, 2004). Astrocytic plasticity is related to glial fibrillary acidic protein (GFAP), an astrocytic cytoskeletal component (Pekny and Pekna, 2004; Nishida and Okabe, 2007; Pekny et al., 2007). In GFAPnegative astrocytes, density of astrocytic processes (Anderová et al., 2001) and hyposmolality-evoked taurine release (Ding et al., 1998) are low, whereas neuronal excitability is high (McCall et al., 1996; Shibuki et al., 1996). In contrast to the genetic GFAP deficiency, GFAP is also involved in neurotrauma, brain ischemia, and neurodegenerative diseases. Under these conditions, astrocytes are reactive, accompanied by hypertrophy of astrocyte processes and upregulation of GFAP (Pekny et al., 2007). GFAP is molecularly associated with excitatory amino acid transporter 1 (EAAT1) [or glutamate/aspartate transporter (GLAST)] and hydrogen/sodium exchanger modulating proteins (Sullivan et al., 2007). GFAP is essential in retaining EAAT1 in the plasma mem-

Received Sept. 29, 2008; revised Nov. 21, 2008; accepted Dec. 19, 2008.

This work was supported by National Institutes of Health Grant NS009140 and University of California, Riverside, College of Natural and Agricultural Sciences. We thank Dr. K. Bauer for astrocyte-specific dipeptide.

Correspondence should be addressed to Glenn I. Hatton, Department of Cell Biology and Neuroscience, University of California, Riverside, CA 92521. E-mail: glenn.hatton@ucr.edu.

Y.-F. Wang's present address: Department of Cellular Biology and Anatomy, Louisiana State University Health Sciences Center, 1501 Kings Highway, Shreveport, LA 71130-3932. E-mail: ywang4@|suhsc.edu.

DOI:10.1523/JNEUROSCI.4669-08.2009

Copyright $\odot 2009$ Society for Neuroscience $\quad$ 0270-6474/09/291743-12\$15.00/0 branes of astrocytes after a hypoxic insult (Sullivan et al., 2007); loss of GFAP results in decreases in the expression and function of GLAST and EAAT2 in astrocytes (Hughes et al., 2004; Li et al., 2008). Thus, GFAP cannot only determine astrocytic morphology but also localize astrocytic functional proteins.

In the hypothalamus, hyperosmotic challenges and lactation cause retraction of astrocytic processes (Hatton, 2004). Retraction of astrocytic processes during lactation may facilitate synchronized activation of oxytocin (OT) neurons during suckling (Hatton, 2004), which determines a bolus release of OT and triggers milk ejections. Controversially, disabling retraction of astrocytic processes by removal of polysialic acid from the neural cell adhesion molecule during pregnancy did not influence OT neuronal activity during suckling (Catheline et al., 2006). Although this finding suggests that chronic retraction of astrocytic processes is not important in astrocytic modulation of neuronal activity in this particular system, it also suggests that some compensatory mechanisms (Pierre et al., 2001; Theodosis et al., 2004) are enough to replace this astrocytic functions in chronic processes. However, this experiment presents neither GFAP involvement nor acute effects of astrocytic plasticity in the modulation of OT neuronal activity. Recently, we have determined that brief suckling can also cause apposition of magnocellular neurons in the supraoptic nucleus (SON), likely resulting from retraction of astrocytic processes (Wang and Hatton, 2007b). It remains unknown whether or not this type of acute astrocytic plasticity has a causal relationship with OT neuronal activity. 
For this purpose, we used OT neurons in the SON in lactating rats as a model to study effects of suckling stimulation on GFAP plasticity and its interactions with OT neuronal activity. The results showed that disabling astrocytic plasticity blocked the milkejection reflex (MER) and reduced neuronal excitability and clustered EPSCs in OT neurons. Suckling initially caused decreases in GFAP expression, which then reversed and became increases that were associated with changes in local neurochemical environment. GFAP was molecularly and dynamically associated with several functional astrocytic proteins. These results are the first to reveal dynamic interactions between GFAP plasticity and neuroendocrine neuronal activity during acute physiological processes.

\section{Materials and Methods}

All procedures in the animal experiments were in accordance with the guidelines on the use and care of laboratory animals set by National Institutes of Health and approved by the Institutional Animal Care and Use Committee of the University of California, Riverside.

Drugs, antibodies, and agents used. All drugs were from Sigma except as otherwise noted. All antibodies were products of Santa Cruz Biotechnology except rabbit polyclonal antibody against aquaporin 4 (AQP4) (Alomone Labs) and a mouse monoclonal antibody from Sigma. Bauer Peptide ( $\beta$-Ala-Lys- $\mathrm{N}_{\varepsilon}$-AMCA), which is actively taken up by astrocytes, was provided by Dr. K. Bauer (Max-Planck-Institut für experimentelle Endokrinologie, Hannover, Germany). Protein G agarose beads were from Millipore. Alexa Fluor 488-, 555-, or 647-labeled secondary antibodies were from Invitrogen. All reagents for Western blots were from GE Healthcare.

In vivo drug application. Adult female Sprague Dawley rats were used on lactating days $8-12$. In suckling experiments, dams were separated overnight from litters of 10 pups but one. On the following morning, animals were anesthetized with urethane ( $1.2 \mathrm{~g} / \mathrm{kg}$ bodyweight, i.p.) and placed in a stereotaxic frame according to a rat brain atlas (Paxinos and Watson, 1998). Body temperature was maintained at $36.5-38^{\circ} \mathrm{C}$, and a femoral vein was cannulated. After exposure of the dorsal surface of the cortex, ligation, and removal of the superior sagittal sinus, an intracerebroventricular guide cannula was implanted, via the dorsal approach, as described previously (Okere et al., 1996). Three hours after the operation, gliotoxins, L-aminoadipic acid (L-AAA), or fluorocitrate (sodium salt) was microinjected into the cerebroventricular system. In preparing the L-AAA solution, $10 \mathrm{~mm}$ was dissolved in an artificial CSF (aCSF) by heating and sonication immediately before application. After $1-2 \mathrm{~h}$, suckling stimulation was applied for $1 \mathrm{~h}$, and the MER and litter bodyweight gains were observed.

Samples of brain tissues. SON samples were collected for both in vivo and in vitro experiments. In suckling experiments, lactating rats were separated from all 10 pups for $4 \mathrm{~h}$ and then divided into the following three groups: non-suckling group, suckling group (suckling for 5-10 min before the first MER), and MER group (suckling until the third or fourth milk ejections occurred). After decapitation, the brains were quickly removed (within $30 \mathrm{~s}$ ) and immersed in oxygenated, ice-cold slicing solution (one-thirds 10\% sucrose plus two-thirds regular aCSF) for 1-2 min, to stop metabolic activity. The regular aCSF contained the following (in mm): $126 \mathrm{NaCl}, 3 \mathrm{KCl}, 1.3 \mathrm{MgSO}_{4}, 2.4 \mathrm{CaCl}_{2}, 1.3 \mathrm{NaH}_{2} \mathrm{PO}_{4}, 26 \mathrm{NaHCO}_{3}$, 10 glucose, and 0.2 ascorbic acid, $\mathrm{pH} 7.4$ adjusted with 3-[Nmorpholino] propanesulfonic acid $(\sim 2 \mathrm{~mm})$ and was gassed with $95 \%$ $\mathrm{O}_{2} / 5 \% \mathrm{CO}_{2}$. Brains were fixed in $4 \%$ paraformaldehyde solution for $72 \mathrm{~h}$ at $4^{\circ} \mathrm{C}$, then cut into $40-\mu \mathrm{m}$-thick sections, and subjected to immunostaining. In separate groups of rats, brains were sliced $(200 \mu \mathrm{m})$; SONs were punched out of slices and lysed for Western blots immediately. To reduce the variability of results from different slices/sections, punches of equivalent size and location in the SON were assigned to different treatment groups.

To obtain in vitro samples for studying mechanisms underlying GFAP plasticity, SONs were sampled in rats. Coronal hypothalamic slices, 200 $\mu \mathrm{m}$ thick, were sectioned and preincubated at room temperature (RT) $\left(21-23^{\circ} \mathrm{C}\right)$ for $1 \mathrm{~h}$ in oxygenated aCSF. Slices were then treated with different drugs before being fixed for immunocytochemistry or lysed for Western blots as described in Results.

Immunocytochemistry. In observing effects of suckling on GFAP expression, immunocytochemistry was performed as described previously (Hatton et al., 1987; Wang and Hatton, 2007b) with minor modification. In brief, hypothalamic slices containing the SON were permeated with $0.3 \%$ Triton X-100 for $30 \mathrm{~min}$, and nonspecific binding sites to antibodies were blocked by incubation of the sections in $0.3 \%$ gelatin for $30 \mathrm{~min}$. The slices were incubated with antibodies against OT and vasopressin neurophysins (NPs) (goat, 1:400 dilution from $200 \mu \mathrm{g} / \mathrm{ml}$ stock) and against GFAP (mouse, 1:300) or AQP4 (rabbit, 1:300) overnight at $4^{\circ} \mathrm{C}$ and then incubated with donkey antibodies against goat (Alexa Fluor 647 labeled, 1:1000), mouse (Alexa Fluor 488 labeled, 1:1000), and rabbit (Alexa Fluor 555 labeled, 1:1000) for 1.5 h at RT. Finally, Hoechst (1:500 for $15 \mathrm{~min}$ ) was added to label the nuclei in all samples except the groups of slices that were preloaded with Bauer Peptide $\left(20 \mu \mathrm{M}, 35^{\circ} \mathrm{C}, 2 \mathrm{~h}\right)$. Sections were examined with a laser scanning confocal microscope (Leica TCP SP2) in sequential scanning mode, single- and $Z$-series sectioned ( $0.5 \mu \mathrm{m}$ thick for each section).

Western blots and immunoprecipitation. The methods for protein experiments were performed as reported previously (Wang and Hatton, $2007 a, b)$. In brief, SON lysates were sonicated and centrifuged to remove insoluble components. Concentration of proteins was assayed using BCA protein assay reagents of Pierce and Synergy 2 plate reader (BioTek Instruments). In each treatment, $60 \mu \mathrm{g}$ of protein was loaded and separated on $10 \%$ SDS-PAGE gels. Protein was transferred onto polyvinylidene difluoride (PVDF) membranes at $4^{\circ} \mathrm{C}$. After pretreatment with $5 \%$ milk solids for $1 \mathrm{~h}$ at RT, protein membranes were then incubated with antibodies against GFAP (mouse or goat, 1:500, overnight at $4^{\circ} \mathrm{C}$ ), rabbit actin (1:500, $4 \mathrm{~h}$ at RT), rabbit total extracellular signal-regulated protein kinase $2\left(1: 1000,1 \mathrm{~h}\right.$ at RT), or mouse tubulin $\left(1: 500\right.$, overnight at $\left.4^{\circ} \mathrm{C}\right)$, respectively. Protein bands were visualized on the second day using horseradish peroxidase-conjugated secondary antibodies and an enhanced chemiluminescence system. Stripping of membranes and reprobes with different antibodies were applied when two target proteins having similar molecular weight were examined. All data were collected at least in triplicate.

In immunoprecipitation, total lysates were precleared with protein $G$ agarose beads to reduce nonspecific binding. Immunoprecipitating antibody (mouse GFAP, $1.5 \mu \mathrm{g}$ ) was added to SON lysates (1000-1500 $\mu \mathrm{g} / 500 \mu \mathrm{l})$ to form an immunocomplex. After overnight incubation at $4^{\circ} \mathrm{C}$, the immunocomplexes were captured by adding $50 \mu \mathrm{l}$ of protein $\mathrm{G}$ agarose bead slurry and gently rocking for $2 \mathrm{~h}$ at $4^{\circ} \mathrm{C}$. The agarose bead immunocomplexes were collected by a pulse centrifugation. After discarding the supernatant and washing the beads three times with ice-cold buffer, the agarose beads were resuspended in $50 \mu$ l of $2 \times$ sample buffer and mixed gently. Beads were boiled for $10 \mathrm{~min}$ to dissociate the immunocomplex. The beads were removed after centrifugation, and the supernatant was run on $10 \%$ SDS-PAGE gels. Protein was then transferred to PVDF membranes. Target proteins on the membrane were detected using Western blots as indicated in Results.

In vitro electrophysiology. The patch-clamp recordings were the same as described previously (Wang and Hatton, 2007a,b). Briefly, 300- $\mu \mathrm{m}-$ thick coronal brain slices containing the SON were obtained from lactating rats. Whole-cell patch-clamp recordings were obtained from magnocellular neurons under visual guidance through an upright microscope. Patch-pipette filling solution contained the following components (in mM): $145 \mathrm{~K}$-gluconate, $10 \mathrm{KCl}, 1 \mathrm{MgCl}_{2}, 10 \mathrm{HEPES}, 1 \mathrm{EGTA}, 0.01 \mathrm{CaCl}_{2}$, $2 \mathrm{Mg}$-ATP, and $0.5 \mathrm{Na}_{2}$-GTP, $\mathrm{pH} 7.3$, adjusted with $\mathrm{KOH}$. In the recordings, $0.05 \%$ Lucifer yellow $\left(\mathrm{K}^{+}\right.$salt $)$was added to the pipette solution to mark the recorded neurons. An Axopatch 200B amplifier (Molecular Devices) was used to collect electrical signals that were filtered and sampled at $5 \mathrm{kHz}$ by Clampex 10 software through a 1320 analog-to-digital/ digital-to-analog converter (Molecular Devices). Data were stored in a computer for offline analysis.

Data collection and analysis. Data analysis for immunocytochemistry, Western blots, and patch-clamp recordings have been described previously (Wang and Hatton, 2007a,b) with minor modification. In evaluat- 
ing GFAP expression in confocal images, the fluorescence intensity in each channel was normalized to a standard curve (1-256) to allow for comparison (and averaging) of separate experiments. The background level was set as 1 through minimum baseline correction using Leica LCS Lite software. ANOVA, paired or nonpaired $t$ test, two-sample Kolmogorov-Smirnov test, Wilcoxon's rank test, and binormial test were used for statistical analyses when appropriate, as instructed by SigmaStat program, and $p<0.05$ was considered significant. When large variances occurred, square-root or log transformations were applied to minimize the interference of individual data with the evaluation of significance levels. All measures were expressed as mean \pm SEM in the percentage of controls, except as otherwise indicated in Results.

\section{Results}

The relationship between astrocytic plasticity in the SON and suckling-evoked MER was first examined in whole animals. The interaction between astrocytes and OT neurons, a prerequisite for the MER, was then linked to GFAP plasticity through examination of EPSCs, GFAP images, and GFAP proteins. The expression of GFAP was further associated with the neurochemical events surrounding OT neurons in brain slices by simulating OT increases and transient $\mathrm{K}^{+}$increases that occur at different stages of suckling. Finally, the functional basis of GFAP involvement in astrocytic modulation of OT neuronal activity was explored using coimmunoprecipitation.

\section{Dependence of OT neuronal activity on astrocytic plasticity}

A common method to evaluate astrocytic participation in neuronal activity is to disable astrocytic functions using gliotoxins. L-AAA can inhibit glutamine synthetase (Glu-Syn) and $\mathrm{Na}^{+}$dependent glutamate reuptake in astrocytes (Pannicke et al., 1994) and effectively influence neuronal activity within $30 \mathrm{~min}$ (Baudoux and Parker, 2008). In the SON, the specificity of L-AAA on astrocytes has not been evaluated. Therefore, we first observed the effects of L-AAA on glutamine-modulated GFAP and Glu-Syn in brain slices in immunocytochemistry. We found that glutamine $(0.1 \mathrm{~mm}, 15 \mathrm{~min})$ significantly increased the expression of GFAP and Glu-Syn in the SON; this effect of glutamine was suppressed by pretreatment of slices with L-AAA (0.25 mM, $30 \mathrm{~min})$ (data not shown). Because both GFAP and Glu-Syn are astrocytic markers, the blocking effects of L-AAA on glutamine actions support a direct action of L-AAA on supraoptic astrocytes. Based on this finding, we observed effect of intracerebroventricular application of L-AAA $(10 \mathrm{mM}, 1 \mu \mathrm{l})$ on the MER. In six lactating rats, none of them showed the MER during $1 \mathrm{~h}$ suckling of 10 pups after L-AAA. Similar to the effect of L-AAA, application of fluorocitrate (100 mM, $1 \mu \mathrm{l}, 2 \mathrm{~h}$ ), another gliotoxin, also blocked the MER during 1 h suckling $(n=3)$. However, the control rats with intracerebroventricular aCSF application $(n=9)$ all showed normal MER, as indicated by simultaneous stretching responses of pups during milk ejections. Together, the litter bodyweight gains $(14.9 \pm 2.5 \%$ of the control) were significantly low $(p<0.05)$ in rats receiving gliotoxins after $1 \mathrm{~h}$ suckling. Because MER results from suckling-evoked synchronized activation in burst firing of OT neurons in the SON and paraventricular nuclei (Belin and Moos, 1986), the inhibitory effect of intracerebroventricular L-AAA suggests widespread inhibition of OT neuronal activity.

Because direct targets of L-AAA were not localized by intracerebroventricular drug applications, we further tested the effects of L-AAA on SON neuronal activity in patch-clamp recordings from hypothalamic slices. L-AAA $(0.25-1 \mathrm{mM}, 10-30 \mathrm{~min} ; n=$ 14) significantly reduced SON neuronal firing activity (Fig. 1A) in a dose-dependent manner. High levels of L-AAA (1 mM) often (seven of nine recordings) caused disruption of the stability of whole-cell configuration after $10 \mathrm{~min}$ perfusion. Low levels of L-AAA $(0.25 \mathrm{~mm})$ or fluorocitrate $(1 \mathrm{~mm})$ similarly reduced the firing rate of SON neurons $(4.0 \pm 0.9$ vs $2.0 \pm 0.7 \mathrm{~Hz} ; p<0.05$; $n=5)$. Similarly, L-AAA also significantly influenced spontaneous EPSCs. High levels of L-AAA (1 mM) completely suppressed EPSCs after 10-20 min perfusion (Fig. 1B1), whereas low levels $(0.25 \mathrm{~mm})$ slowly but persistently reduced the frequency of tonic EPSCs (Fig. 1B2). After pretreatment of slices with L-AAA, OT did not significantly influence the frequency and amplitude of EPSCs (Fig. 1C), suggesting that OT may influence EPSCs in a manner similar to L-AAA.

In previous studies (Wang and Hatton, 2004, 2007a), we found that burst generation was associated with clustered EPSCs. If astrocytic plasticity is associated with bursts/MER, L-AAA may also influence this patterned EPSC activity. The clusters (Fig. $2 A 1)$ were composed of long-duration events (1.0-9 ms at halfwidth) but not those short-duration events $(0.3-1.5 \mathrm{~ms}$ at halfwidth), both of which existed in tonic EPSCs. OT (10 pM, $30 \mathrm{~min})$ significantly increased the instantaneous frequency but decreased EPSC amplitude in the clusters (Fig. 2A2,B). Compared with the control (Fig. $2 A 1, B)$, during L-AAA application $(0.25 \mathrm{~mm}, 30$ $\mathrm{min}$ ), the duration of EPSC clusters (Fig. $2 A 3$ ) and the half-width of EPSCs ( $4.5 \pm 0.6 \mathrm{~ms}$ in control vs $2.2 \pm 0.4 \mathrm{~ms}$ in L-AAA) were reduced significantly, whereas their amplitude and frequency, and rise and decay time constants (tau) remained unchanged (Fig. $2 B$ ). In the presence of L-AAA, the effects of OT on the clusters were reduced significantly (Fig. $2 A 4$ and 2B), indicating involvement of astrocytes in both spontaneous and OT-evoked EPSC clustering. To remove the influence of neuronal firing activity on EPSCs, TTX was applied. In the presence of TTX (Fig. $2 A 5, B)$, clustered EPSCs could still be observed, but the amplitude and instantaneous frequency were lower and rise tau was slower than in controls. Analysis of cumulative probability of amplitude distribution of these miniature EPSCs in the clusters showed a clear separation of the curve of clusters from that immediately before the event $(n=24 ; p<0.05)$, with significantly high amplitude and frequency in the clusters. This result indicates that the generation of clustered EPSCs involves both presynaptic and postsynaptic neurons. In the presence of TTX (Fig. $2 A 6, B)$, the amplitude and instantaneous frequency were lower, frequency was higher, and rise tau became slower in response to OT compared with OT actions only (Fig. $2 B$ ). This result, together with the effect of TTX only, suggests that firing activity has a facilitatory effect on amplitude and instantaneous frequency but an inhibitory effect on intraclustering frequency of the clusters. It is also possible that some extrasynaptic glutamate release was involved after blocking action potentials. After pretreatment of slices with L-AAA (Fig. 2A7), TTX failed to further reduce the cluster amplitude $(35.8 \pm 5.2$ to $15.9 \pm 0.6$ and $35.5 \pm 3.5 \mathrm{pA}$ in control, TTX, and TTX plus L-AAA, respectively; $n=17,24$, and 4 , respectively), although the duration $(39.1 \pm 11.7$ vs $11.2 \pm$ $3.1 \mathrm{~s})$ of clusters and their rising tau (11.6 $\pm 2.2 \mathrm{vs} 5.8 \pm 1.0 \mathrm{~ms})$ were shortened significantly $(p<0.01)$ in the presence of L-AAA. These changes are summarized in Figure $2 B$. These results indicate that TTX did not interrupt the clustered features of EPSCs modulated by L-AAA; L-AAA likely removed extrasynaptic release of glutamate. In the other words, astrocytes played a dominant role in shaping the features of clustered EPSCs, although firing activity was also involved. Finally, pretreatment of slices with L-AAA for 7-20 h significantly reduced or even completely removed EPSCs; no clustered EPSCs were observed in the control (L-AAA treatment only; $n=14)$ or in response to OT $(n=7)$. 
From these results, we tentatively believe that astrocytic plasticity is the final source of tonic and clustered EPSCs.

\section{Rapid changes in GFAP plasticity} during suckling

The observed influences of L-AAA on OT neuronal activity were not likely via its inhibition of glutamate transporters (Pannicke et al., 1994). Inhibition of glutamate transporters can increase extracellular ambient glutamate and enhance EPSCs, at least at its initial actions. However, L-AAA did not increase excitatory synaptic transmission but decreased it. Although this effect could partially result from decreased activity of Glu-Syn and lack of supplement of glutamine to neurons, the changes in clustered EPSCs by L-AAA, e.g., short duration, quick rise tau, and higher amplitude in the TTX, suggest involvement of astrocytic functions in addition to glutamate metabolism. This view is in agreement with the finding that L-AAA disruption of neuronal activity is not attributable to lack of ambient glutamate (Baudoux and Parker, 2008), at least in a short time course.

In previous work, we found that suckling changed astrocytic morphology (Wang and Hatton, 2007b), which is usually associated with GFAP plastic changes (Weinstein et al., 1991). Thus, we further observed effects of intracerebroventricular application of L-AAA and fluorocitrate on GFAP expression in the SON. The result showed that L-AAA ( $10 \mathrm{~mm}, 1 \mu \mathrm{l}, 1 \mathrm{~h} ; n=$ 3 ) reduced GFAP levels $>20 \%$ in confocal images compared with the rats receiving intracerebroventricular aCSF only (Fig. $2 C 1)$. Similar results to L-AAA were also observed after intracerebroventricular application of fluorocitrate $(100 \mathrm{~mm}, 1 \mu \mathrm{l}$, $2 \mathrm{~h}$ ), which also markedly reduced GFAP levels. To examine whether these gliotoxins acted directly on astrocytes in the SON, we further tested effects of L-AAA $(0.25$ $\mathrm{mM}, 30 \mathrm{~min}$ ) on GFAP expression in the brain slices (Fig. 2C2). L-AAA treatment significantly $(p<0.05 ; n=7)$ reduced GFAP staining in the SON $(82.5 \pm 7.3 \%$ of control), indicating that GFAP expression in the SON was involved in the disruption of the MER by L-AAA.

If GFAP plasticity is involved in astrocytic modulation of OT neuronal activity, suckling should also change GFAP expression in the SON. Thus, we further examined GFAP expression in the SON during suckling with immunocytochemistry and Western blotting. As shown in Figure $3 A 1$, suckling for 5-10 $\mathrm{min}$ caused a general reduction of GFAP
A

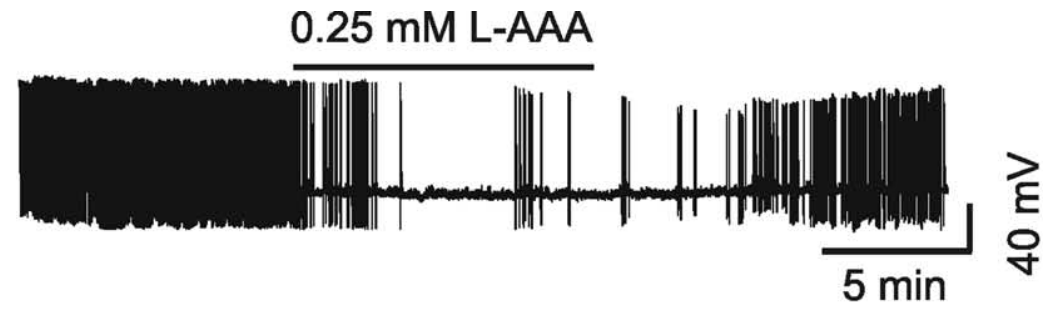

B

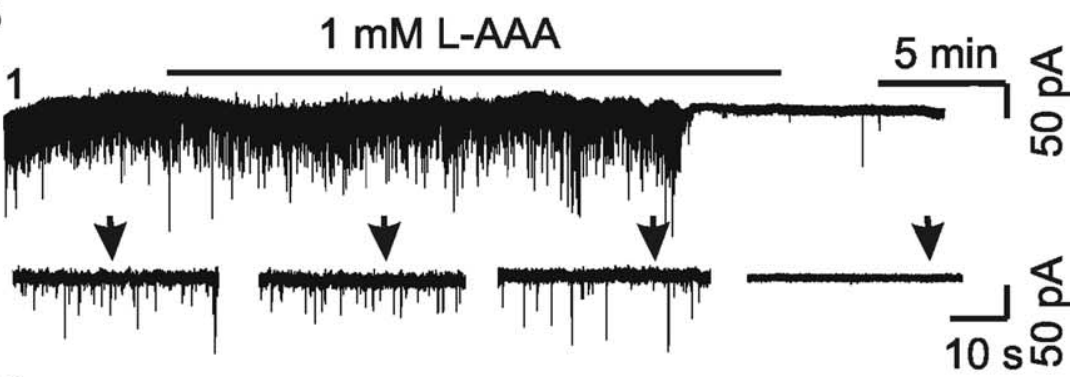

2

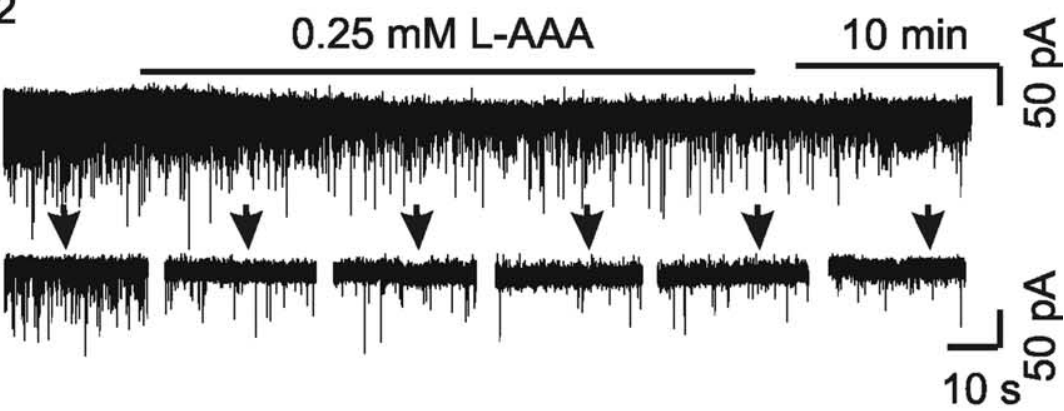

C

1
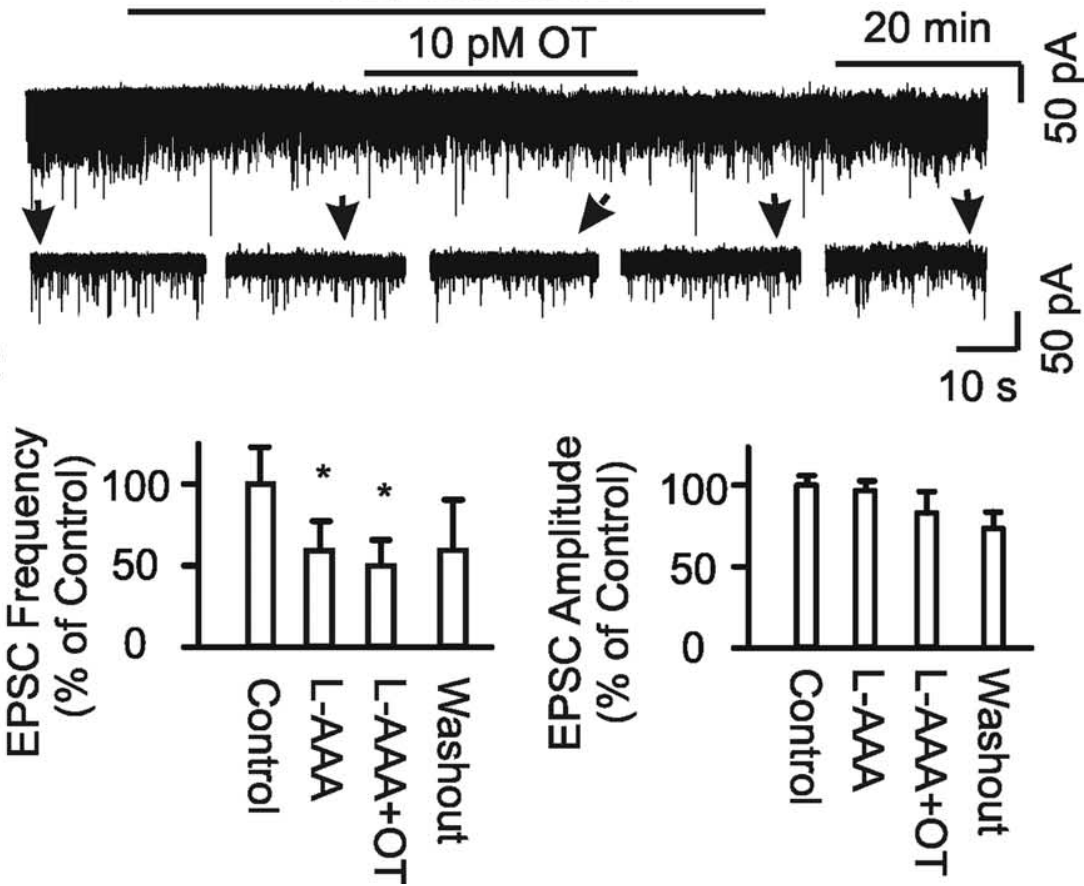

Figure 1. Effects of disabling astrocytic functions on OT neuronal activity in lactating rats. $\boldsymbol{A}-\boldsymbol{C}$, Whole-cell patch-clamp recordings in brain slices containing SON. $\boldsymbol{A}$, Exemplary current-clamp recording showing firing activity before, during, and after bath application of L-AAA $(0.25 \mathrm{~mm})$ at initial membrane potential of $-60 \mathrm{mV}$. $B$, Exemplary voltage-clamp recordings of tonic spontaneous EPSCs at $-70 \mathrm{mV}$. B1, Effects of $1 \mathrm{~mm} \mathrm{~L}-A A A$. B2. Effects of $0.25 \mathrm{~mm} \mathrm{~L}-A A A$. Recordings showing full traces (top) and their expanded episodes (bottom) as indicated by arrows. C, Effects of OT (10 pM) on EPSCs in the presence of L-AAA. C1, Exemplary recording. C2, Summary bar graphs showing percentage changes relative to controls in frequency and amplitude in response to L-AAA and $0 \mathrm{~T}(n=6) .{ }^{*} p<0.05$ compared with the control. 
A

1. Control

2. OT

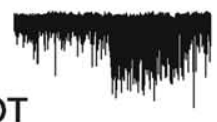
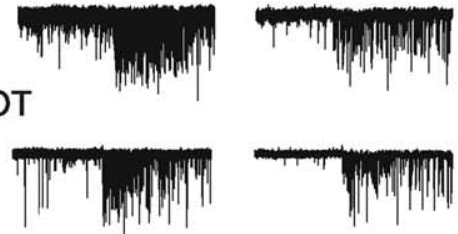

3. L-AAA
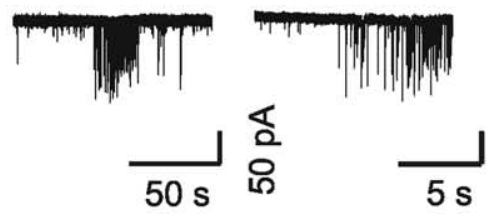

咅

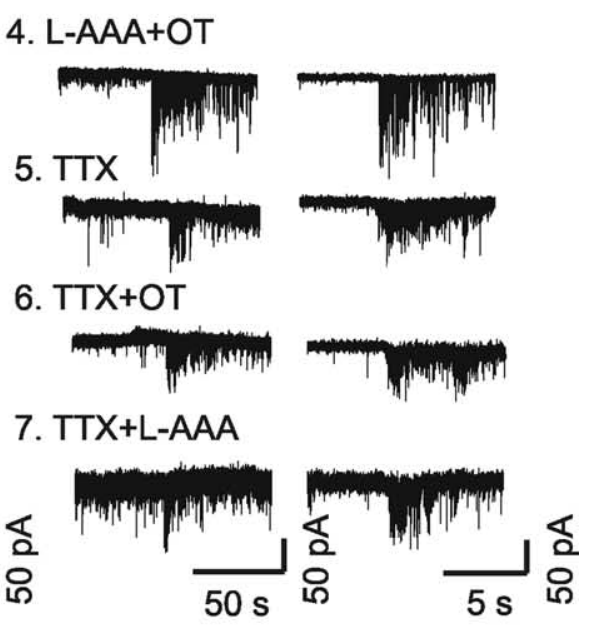

B

1. Amplitude

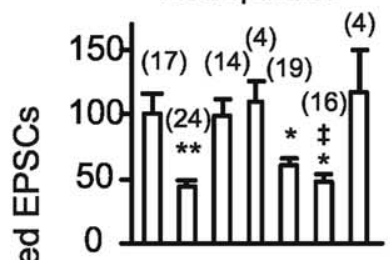

4. Rise tau

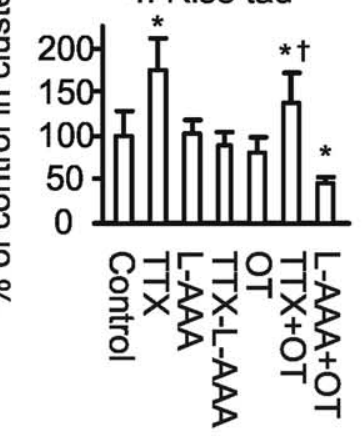

C

1. In vivo

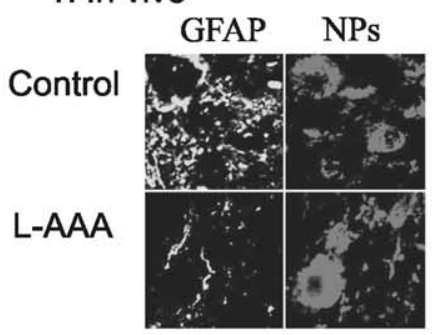

2. Frequency

\section{Duration}

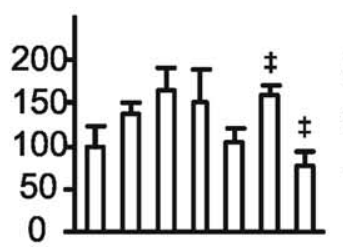

5. Decay tau
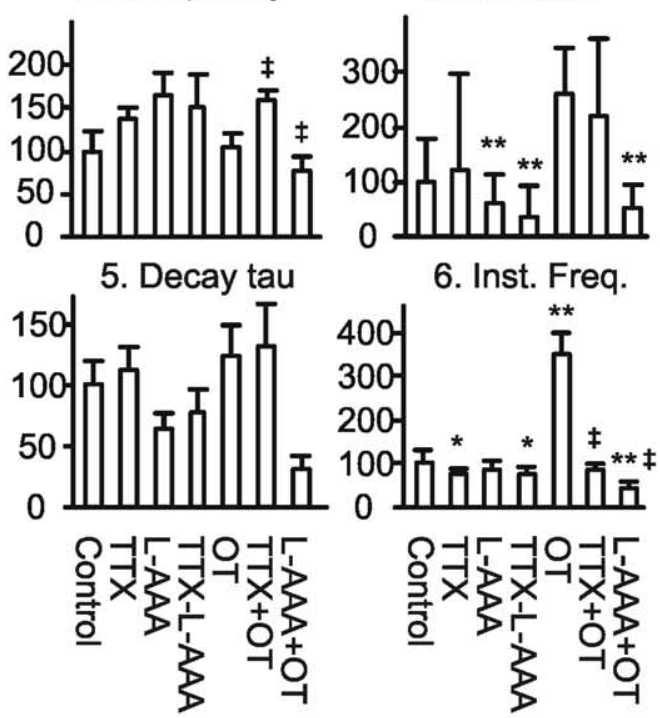

6. Inst. Freq.

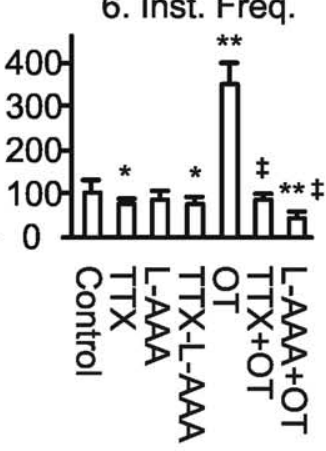

2. In vitro

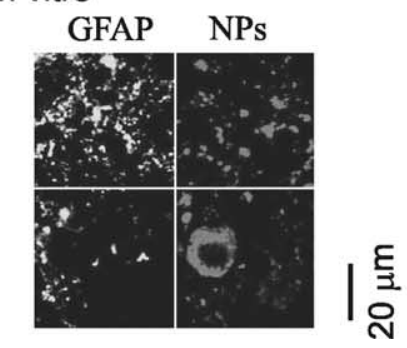

Figure 2. Clustered EPSCS and their sources. $\boldsymbol{A}$, Exemplary clustered EPSCS (left) and their expanded episodes (right) under different conditions in brain slices. Each example is taken from a period of $30 \mathrm{~min}$ recording at a holding membrane potential -70

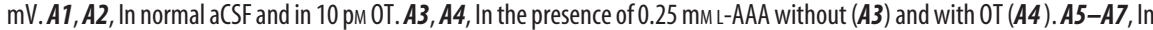
the presence of $0.5 \mu \mathrm{M}$ TTX, TTX plus 10 рм 0T or plus $0.25 \mathrm{~mm} \mathrm{L-AAA}$ on miniature EPSCS. $\boldsymbol{B}$, Bar graphs showing the amplitude (B1), frequency (B2), duration (B3), rise time constant (tau; B4), decay tau (B5), and instantaneous frequency (Inst. Freq.; $\boldsymbol{B}$ 6) of the clustered EPSCs under different conditions as marked in the graphs. ${ }^{*} p<0.05$ and ${ }^{* *} p<0.01$ compared with the control; ${ }^{\dagger} p<0.05$ and ${ }^{\ddagger} p<0.01$ compared with 0 T only. The numbers in parentheses in $\boldsymbol{B} 1$ are that of clustering events. $\boldsymbol{C}$, Confocal images showing immunostaining for GFAP and neurophysins (NPS) of OT and vasopressin. C1, Effect of intracerebroventricular application of L-AAA ( $10 \mathrm{~mm}, 1 \mu \mathrm{l}, 1.5 \mathrm{~h})$. C2, Effects of incubation of brain slice in the presence of L-AAA $(0.25 \mathrm{~mm}, 30 \mathrm{~min})$ on GFAP expression.

staining in the SON in confocal microscopy. The diameters of GFAP filaments decreased, whereas the number of astrocytic somata with stronger GFAP staining increased markedly. These changes were partially reversed immediately after the
MER, i.e., number and intensity of GFAP filaments increased from a low level during initial stage of suckling, whereas their diameters became thicker. GFAP-positive somata remained large, but the intensity of their GFAP staining weakened. Considering that quantification of single scanning images in confocal microscopy may not represent the whole section of the staining, even randomized, we further verified the GFAP plasticity using $Z$-series scanning (Fig. $3 A 2$ ) and fluorescence microscopy (Fig. $3 A 3)$. Consistently, all the three methods yielded similar results. Because $Z$-stacks went through all sections of a GFAP filament and fluorescence microscopy revealed the sum of GFAP staining in all sections, the results strongly support the findings of single-scanning-based analysis of the confocal microscopic images (Fig. 3A4). Consistent with confocal images, analyzing GFAP proteins in Western blots showed that suckling significantly decreased GFAP protein levels in the SON, which partially recovered after the MER, i.e., biphasic or reversible. Interestingly, the partial reversal of GFAP levels at $50 \mathrm{kDa}$ bands at the MER (Fig. 3B1) was accompanied by significant increases in the small fragments of GFAP (Fig. 3B2). This result suggests that suckling at initial stage might strongly increase depolymerization of GFAP filaments and completely degrade some GFAP molecules, whereas the MER increased both GFAP polymerization and formation of GFAP fragments.

Suckling-reduced GFAP by direct action of OT on astrocytes

Suckling changes the neurochemical environment in the SON (Crowley and Armstrong, 1992), which may influence GFAP plasticity. OT is a critical mediator of suckling-evoked MER (Wakerley et al., 1994) and can cause acute retraction of astrocytic processes (Wang and Hatton, 2007b). Thus, it is likely that OT also mediates suckling-elicited GFAP reduction. To test this hypothesis, OT was applied to slices from lactating rats at different times and doses, and then confocal images of GFAP immunostaining were analyzed (Fig. 4A). To link GFAP with astrocytic morphology, before OT treatment, the slices were loaded with Bauer Peptide, an astrocyte-specific fluorescent dipeptide (Dieck et al., 1999; Espallergues et al., 2007). In response to OT ( $10 \mathrm{pm}, 5 \mathrm{~min}$ ), the Bauer Peptidefilled astrocytic processes became clearer and formed bundles, whereas more Bauer Peptide in astrocytes was accumulated in the somata. As time elapsed (from $5 \mathrm{~min}$ to $30 \mathrm{~min}$ ), Bauer 


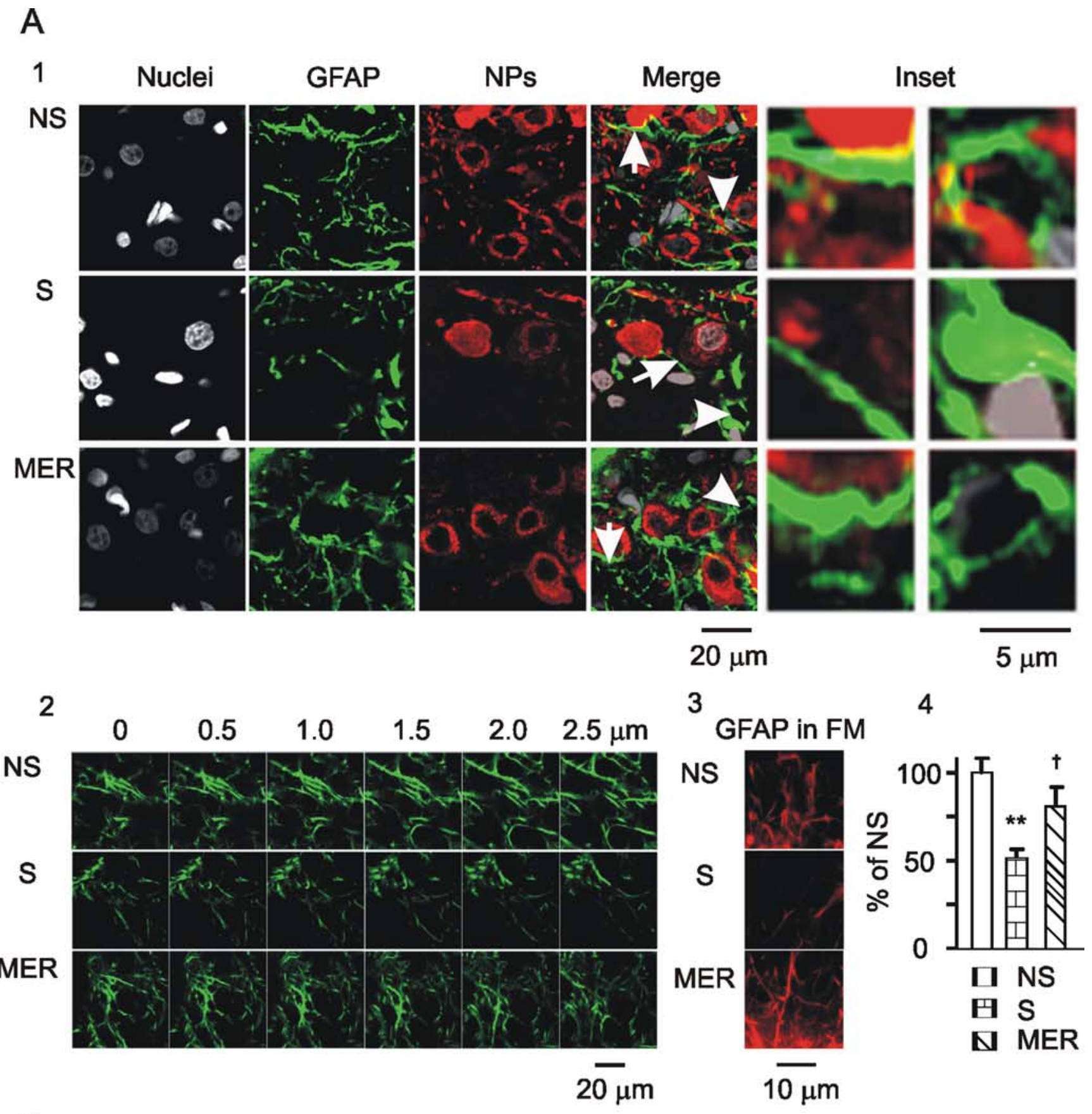

B

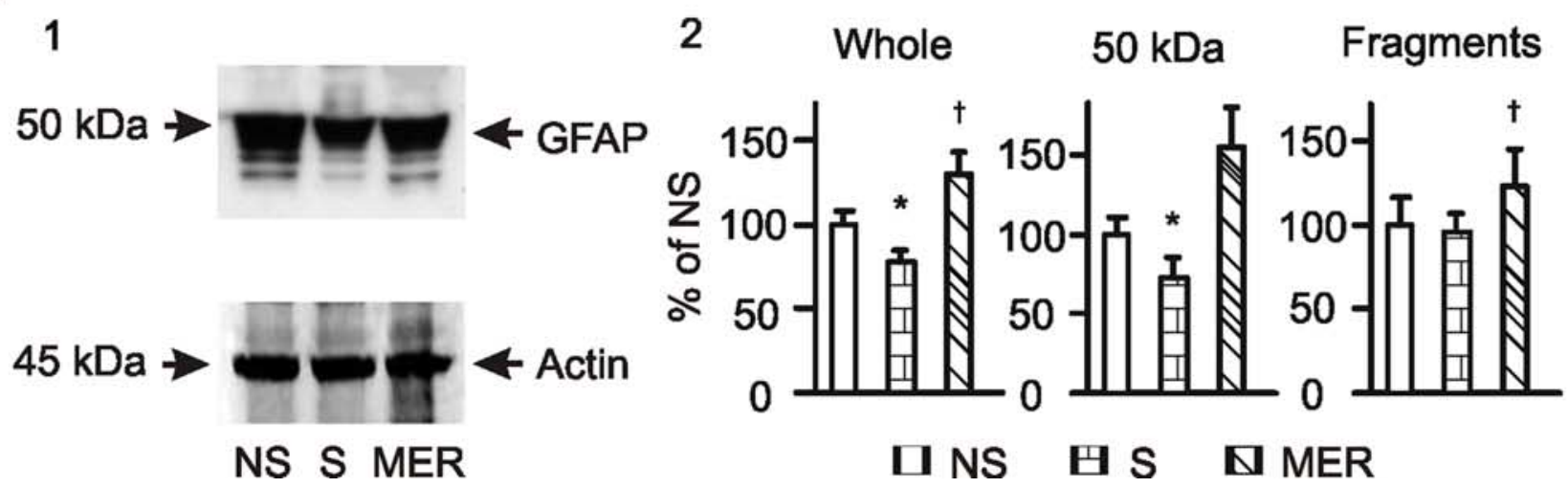

Figure 3. GFAP expression in the SON at different stages of suckling. $\boldsymbol{A}$, Microscopic images. $\boldsymbol{A} 1$, Exemplary images in single-scan model showing nuclei, GFAP, neurophysins (NPS), their merges, and insets (from left to right) in rats, non-suckling (NS), during suckling (S), and immediately after suckling-induced MER. The insets show the special areas in the merge channel indicated by the arrows (GFAP filaments, left insets) or arrowheads (GFAP at somata, right insets). A2, GFAP images in Z-stack model of confocal microscopy with $0.5 \mu$ m distance between (Figure legend continues.) 
Peptide-loaded astrocytic processes reduced in length and width gradually, indicating retraction of functional astrocytic processes. Thus, despite the increased loading in astrocytic somata, astrocytic volume tended to be reduced in response to OT (Fig. 4A2). Before OT treatment, expression of GFAP was loosely compacted in perinuclear areas of astrocytes; GFAP filaments were more aligned, forming clear bundles. OT time dependently reduced GFAP-positive components. In response to 10 pM OT, GFAP expression was slightly but significantly reduced at 5-10 min and further reduced after $30 \mathrm{~min}$ (Fig. $4 A 1, A 2)$. Similar to the temporal effects of small concentration of OT, increasing OT levels from $10 \mathrm{pm}$ to $1 \mathrm{~nm}(n=6)$ dose dependently decreased GFAP expression (Fig. 4A3). The changes in GFAP expression were, in general, consistent with Bauer Peptide uptake in astrocytic processes. However, the dramatically increased somatic size (shown by Bauer Peptide) but not GFAP staining at the somata indicates that GFAP expression can differ from the morphological changes in different microdomains in astrocytes.

In support of GFAP morphological plasticity, additional analysis of GFAP proteins in Western blots showed that OT time and dose dependently modulated GFAP expression. Increasing the time of OT application ( $10 \mathrm{pM}, 0,5$, and $30 \mathrm{~min}$ ) gradually reduced $50 \mathrm{kDa}$ GFAP levels, which did not significantly change the expression of small fragments of GFAP (Fig. 4B1). However, changing the levels of OT ( $30 \mathrm{~min}, 0,10 \mathrm{pM}, 1 \mathrm{~nm}$ ) differentially influenced GFAP protein expression. OT at 10 pM significantly decreased $50 \mathrm{kDa}$ GFAP; at $1 \mathrm{nM}$, OT weakened the decrease of 50 kDa GFAP but increased GFAP small fragments (Fig. 4B2). The results are summarized in Figure $4 B 3$. It is noteworthy that $10 \mathrm{pM}$ OT time dependently reduced GFAP expression in the confocal images and in protein levels. Meanwhile, despite reducing GFAP images, $1 \mathrm{~nm}$ OT restored $50 \mathrm{kDa}$ GFAP and increased GFAP fragments. The effect of high levels of OT on GFAP protein expression was similar to that after the MER. This suggests that high concentration of OT in the SON may occur around the MER and is partially responsible for GFAP expression changes. However, the reversal of GFAP filaments in confocal images was not evoked by OT, suggesting involvement of some other mechanisms in GFAP repolymerization.

Microscopic images cannot distinguish depolymerization of GFAP filaments from degradation of GFAP monomers. To differentiate these two processes, we further observed effects of protease inhibitors on OT-evoked GFAP reduction. Pretreatment of slices with a mixture of protease inhibitors $(0.5 \times$ from $100 \times$ stocking solution; P8340; Sigma) for $30 \mathrm{~min}$ significantly enhanced the intensity of GFAP images ( $132 \pm 5.7 \%$ of control with same solvent as the inhibitors; $p<0.05 ; n=4$ ) but did not significantly influence OT $(0.1 \mathrm{nM}, 30 \mathrm{~min})-$ reduced GFAP expression (Fig. 4C1). This result indicates that OT reduced GFAP levels in microscopic images by promoting GFAP depolymerization. Moreover, in Western blotting (Fig. $4 C 2$ ), the small GFAP fragments disappeared after pretreat-

\section{$\leftarrow$}

(Figure legend continued.) two consecutive sections. A3, GFAP images in fluorescence microscopy (FM) of $400 \times$ amplification. $\boldsymbol{A 4}$, Summary graphs $(n=6)$ for $\boldsymbol{A} 1$ showing relative intensity of GFAP at different stages of suckling. $\boldsymbol{B}$, GFAP expression in Western blots. B1, Exemplary bands from left to right showing GFAP proteins (top) during non-suckling, suckling, and the MER and their corresponding loading controls with actin (bottom). B2, Summary graphs ( $n=$ 3) showing relative amounts of GFAP to the control (NS) in the whole bands, bands at $50 \mathrm{kDa}$ (full size), and their smaller fragments, respectively. ${ }^{*} p<0.05$ and ${ }^{* *} p<0.01$ compared with non-suckling; ${ }^{\dagger} p<0.05$ compared with suckling. Other annotations refer to Figure 2 . ment of the slices with the protease inhibitors with or without OT, indicating the existence of a basal level of protease activity and GFAP degradation in the SON. Together with the changes in confocal images, this result indicates that low levels of OT increase GFAP depolymerization and inhibit GFAP synthesis or remove GFAP antigens, although high levels of OT enhance GFAP assembling and degradation (not simply dissembling) simultaneously.

OT has multiple targets in the SON (Gimpl and Fahrenholz, 2001). Although OT receptors exist on both neurons and astrocytes in the SON (Wang and Hatton, 2006), OT could influence GFAP expression indirectly. Therefore, we further tested effects of OT on GFAP expression after treatment of slices with tetanus toxin (TeTx), an agent that blocks synaptic vesicle release from the OT-secreting system (Halpern et al., 1990; de Kock et al., 2003). As shown in Figure 5A, treatment of slices with $\operatorname{TeTx}(10 \mathrm{~nm}, 2-4 \mathrm{~h})$ almost completely suppressed EPSCs $(4.6 \pm 1.0$ vs $0.4 \pm 0.2$ events/s before and after TeTx; $n=8$ ) and blocked OT-evoked EPSC reduction (Wang and Hatton, 2007a), indicating effective suppression of synaptic vesicle release. In the presence of TeTx, OT application could still reduce GFAP staining (Fig. 5B), which was $70.4 \pm 3.5 \%$ of the control $(p<0.01 ; n=6)$. Thus, OT-evoked GFAP reduction was very likely a direct action of OT on astrocytes.

\section{Suckling-evoked GFAP reexpansion by transiently increasing extracellular $\mathrm{K}^{+}$levels}

Increased OT release in the SON during suckling (Neumann et al., 1993) can explain GFAP reduction before MER occurrence; however, this does not explain the reversal of GFAP images after the MER. The most dramatic neuronal event in the SON during suckling is the synchronized bursting in OT neurons (Belin and Moos, 1986). Synchronized bursts can increase extracellular $\mathrm{K}^{+}$in the SON (Leng and Shibuki, 1987) and in turn promote astrocytic uptake of $\mathrm{K}^{+}$and water (Walz and Wuttke, 1999), facilitating reexpansion of astrocytic processes. To test this hypothesis, we observed effects of transient exposure of the slices to high $\mathrm{K}^{+}(12 \mathrm{~mm}, 30 \mathrm{~s})$ during incubation of the slices with OT $(0.1 \mathrm{nM}, 20 \mathrm{~min})$ on GFAP expression in immunostaining and Western blots. The result showed that the transient high $\mathrm{K}^{+}$stimulation significantly increased GFAP filaments (Fig. 6A1,A2) as well as GFAP proteins (Fig. $6 A 3, A 4)$. This result is consistent with the facts that $\mathrm{K}^{+}$channels are abundant on astrocytes in the SON (Armstrong et al., 2005), and burst firing can activate membrane $\mathrm{K}^{+}$channels on neighboring astrocytes (Gommerat and Gola, 1996).

$\mathrm{K}^{+}$channel activity is closely associated with water transportation. AQP4 (a water channel protein) is coexpressed with $\mathrm{K}^{+}$ channels (Nagelhus et al., 2004); the clearance of high extracellular $\mathrm{K}^{+}$needs AQP4 (Amiry-Moghaddam et al., 2003; Simard and Nedergaard, 2004; Binder et al., 2006). AQP4 is richly expressed in the SON (Badaut et al., 2000) and can be upregulated by activation of V1a receptors (Niermann et al., 2001), a target of OT (Rosso et al., 2002, 2004). If extracellular $\mathrm{K}^{+}$levels are indeed involved in the process of suckling/MER, then AQP4 expression should also show dynamic changes during the suckling. This hypothesis is supported by the following observations. AQP4 was heavily expressed in the SON in lactating rats (Fig. 6 B1). Suckling significantly $(p<0.05 ; n=6)$ reduced AQP4 intensity, which was partially reversed after the occurrence of MER (Fig. 6B2). Consistent with the images, suckling also caused dual changes in AQP4 proteins, i.e., decreases first and then reversal after the MER (Fig. 6B3,B4). Whether the MER-evoked AQP4 reversal 
A

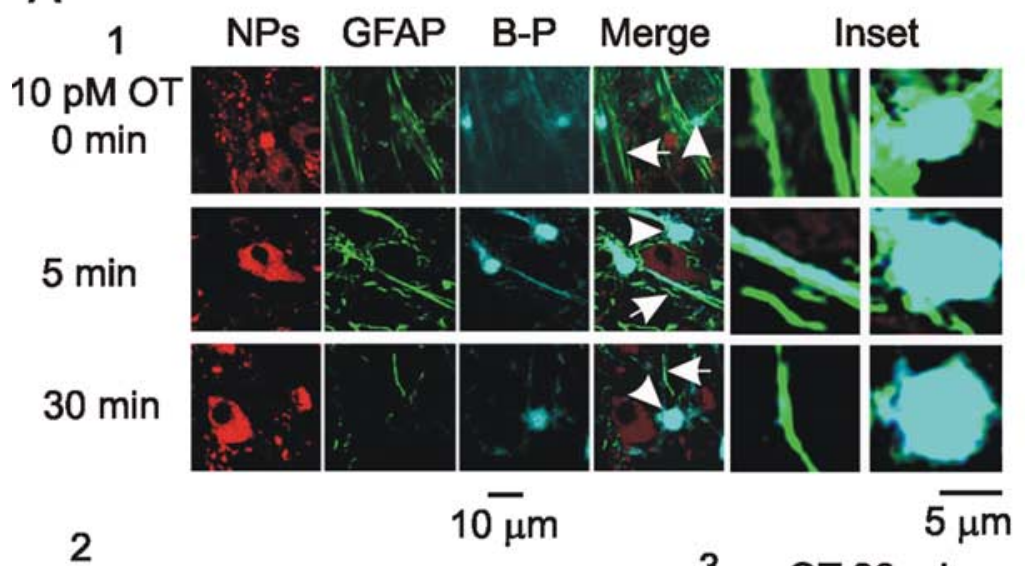

B 1

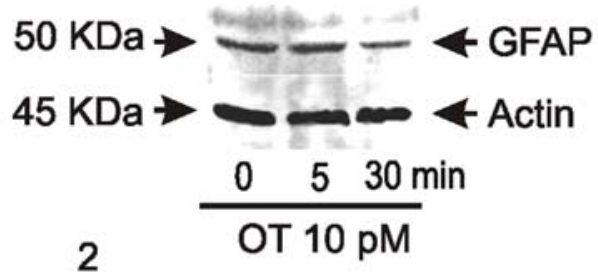

$50 \mathrm{KDa} \rightarrow=-2+\mathrm{GFAP}$

$45 \mathrm{KDa} \rightarrow-3=-4$ Actin

$\frac{010 \mathrm{pM} 1 \mathrm{nM}}{\text { OT } 30 \mathrm{~min}}$

3

Whole $50 \mathrm{KDa}$ Fragments

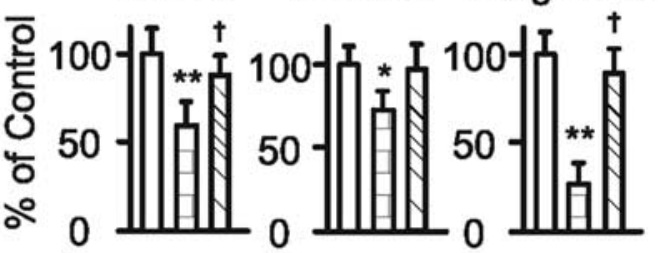

I Control $\mathbf{O} 10 \mathrm{pM} \mathbf{\mathrm { N }} 1 \mathrm{nM}$
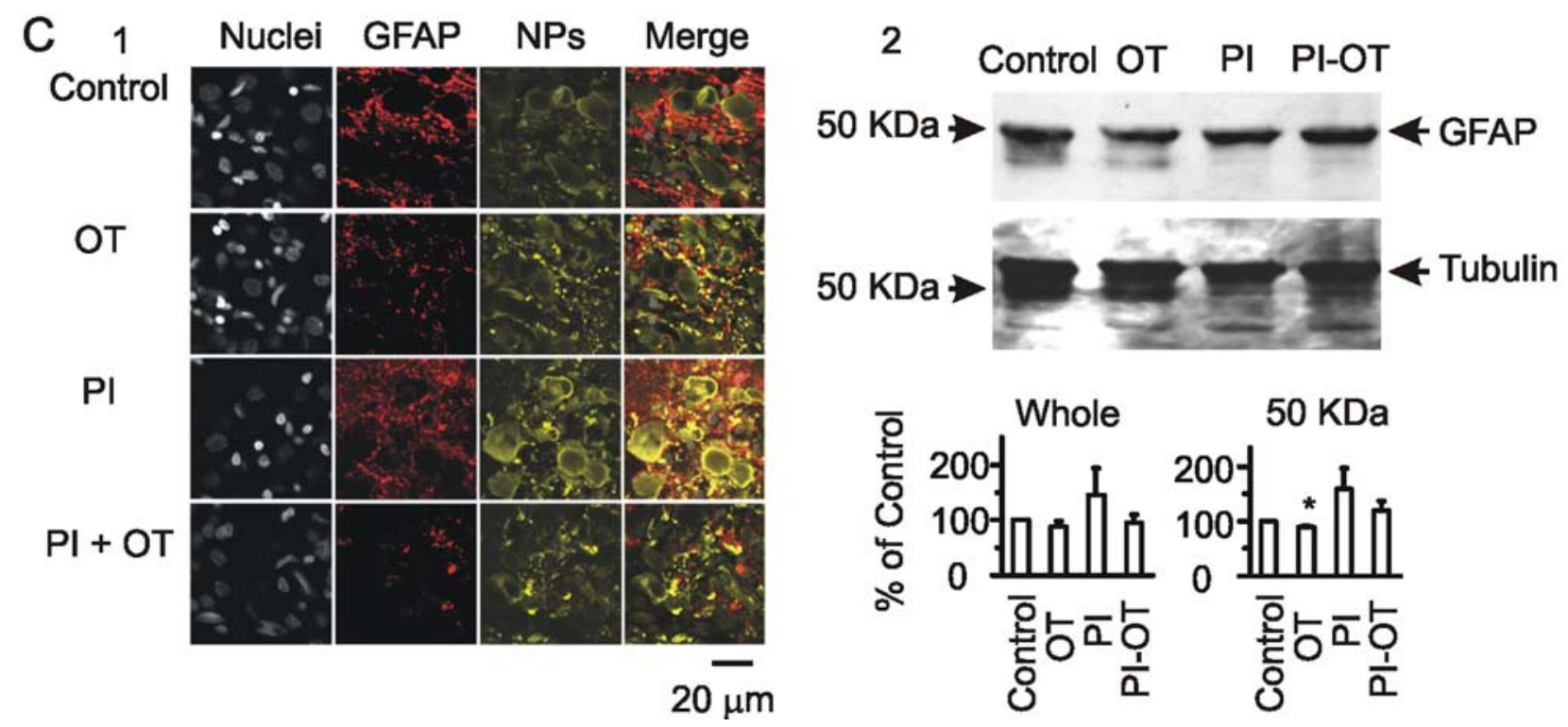

Figure 4. Effects of $0 T$ on astrocytic plasticity in the SON. A, GFAP expression in astrocytes in brain slices. A1, Exemplary images of neurophysins (NPs), GFAP, Bauer Peptide (B-P), their merges, and insets (from left to right) at 0,5 , and 30 min of 0 T treatment. $A 2$, Summary graphs showing relative intensity of Bauer Peptide loading $(n=3)$ and GFAP staining $(n=8)$ relative to the control (0T at $0 \mathrm{~min}$ ). ${ }^{*} p<0.05$ and ${ }^{* *} p<0.01$ compared with $0 \mathrm{~min} ;{ }^{\dagger} p<0.05$ compared with $5 \mathrm{~min}$. A3, GFAP images in response to different concentrations of $0 \mathrm{~T}$ (30 min). $\boldsymbol{B}$, GFAP expression in Western blots. B1, Exemplary bands (from left to right) showing GFAP proteins (top, using mouse antibody from Sigma, G3893) and the loading controls (actin, bottom) at 0,5, and 30 min of 10 pM 0 T treatment. B2, GFAP and actin at $30 \mathrm{~min}$ of $0,10 \mathrm{pm}$, and $1 \mathrm{~nm} 0 \mathrm{~T}$ treatments. $B 3$, Summary graphs $(n=3)$ showing relative amounts of GFAP to the control in the whole bands, bands at $50 \mathrm{kDa}$ (full size), and the smaller fragments, respectively. ${ }^{*} p<0.05$ and ${ }^{* *} p<0.01$ compared with control (0 0T); ${ }^{\dagger} p<0.05$ compared with 10 pm $0 \mathrm{~T}$. C, Effect of protease inhibitors (PI) on $0 \mathrm{~T}$-evoked GFAP expression. C1, Exemplary confocal images showing GFAP expression in control, $0 \mathrm{~T}(0.1 \mathrm{~nm}, 30 \mathrm{~min})$, a mixture of protease inhibitors ( $45 \mathrm{~min}$ ), and protease inhibitors (starting $15 \mathrm{~min}$ before) plus OT (0.1 nм, $30 \mathrm{~min}$ ) as labeled. (2, GFAP expression in Western blots. Top panel shows bands of GFAP (top) and tubulin (loading control; bottom); bottom panel shows graphs ( $n=4)$ summarizing whole GFAP proteins (left) and GFAP at $50 \mathrm{kDa}$ (right). Other annotations refer to Figures 2 and 3.

was achieved by activation of V1a receptors by a higher level of OT remains to be tested. These results support a correlative relationship between GFAP expression and $\mathrm{K}^{+}$and water homeostasis in the SON.
Molecular association of GFAP with astrocytic structural and functional proteins during suckling

GFAP influences neuronal activity by many approaches (Tekkok and Ransom, 2004). Molecular associations between GFAP and as- 
A
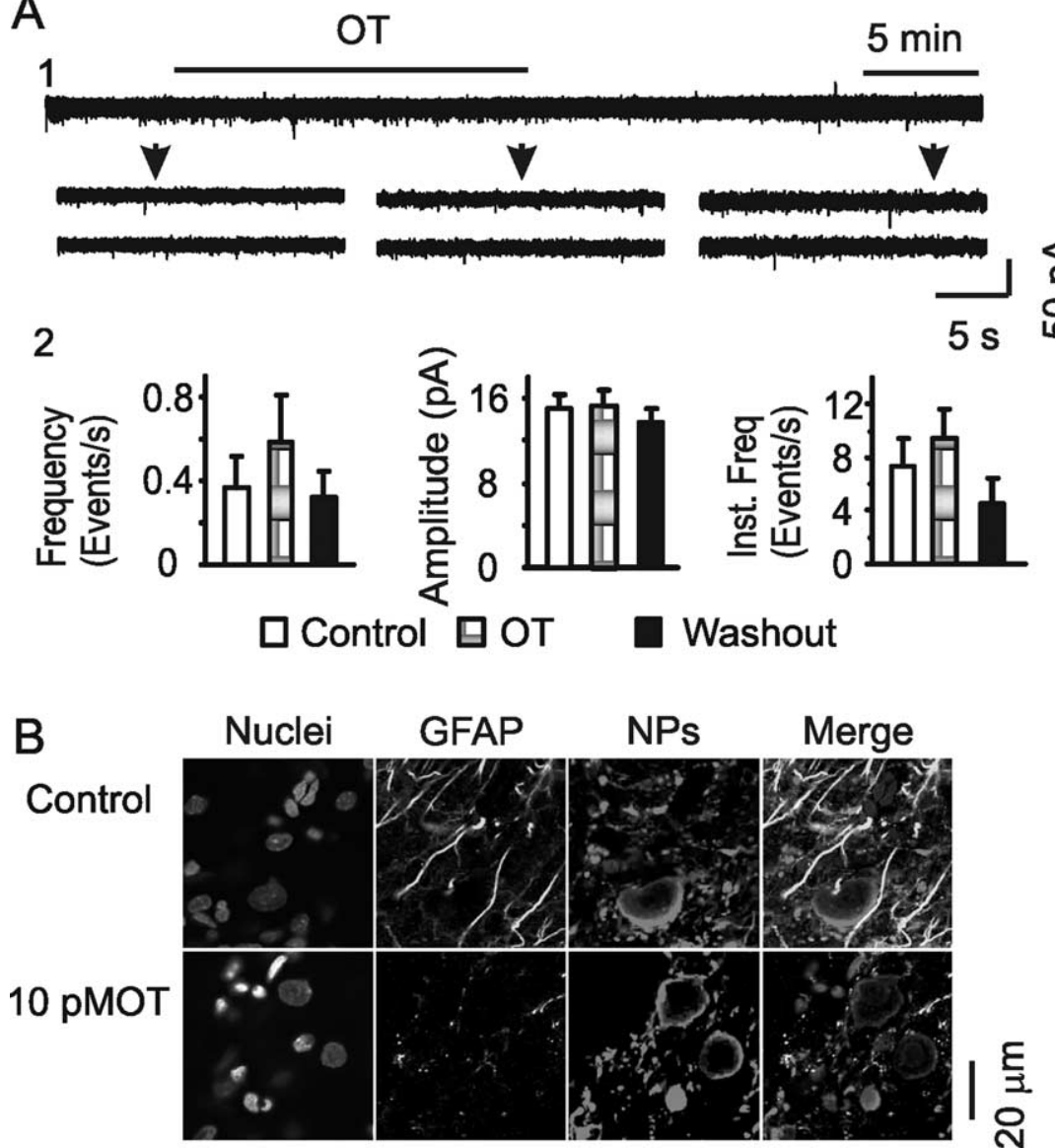

Figure 5. Effects of OT on GFAP expression after blocking synaptic release. $A$, Effects of $0 \mathrm{~T}$ (10 pм) on EPSCs after pretreatment of slices with TeTx (10 nм, 2 h). A1, Exemplary voltage-clamp recordings. A2, Summary graphs $(n=8)$ showing the averaged frequency, amplitude, and instantaneous frequency in control, $0 \mathrm{~T}$, and washout of $0 \mathrm{~T}$. $B$, Confocal images showing effects of $0 \mathrm{~T}$ (10 pm, $30 \mathrm{~min})$ on GFAP expression after the pretreatment of slices with tetanus toxin. Other annotations refer to Figures 1-3.

trocytic functional molecules (Sullivan et al., 2007) are an important approach in astrocytic plasticity. In the SON, it remains unknown whether suckling/OT stimulation influences OT neuronal activity by altering molecular associations between GFAP and astrocyte functional proteins. Thus, we tested the hypothesis that astrocytes influence OT neuronal activity by changing molecular associations between GFAP and other structural and functional molecules of astrocytes.

Among many astrocytic proteins, AQP4, actin, Glu-Syn, and serine racemase are particularly interesting. Burst firing causes astrocytic swelling (Anderová et al., 2001). The MER was accompanied by expression of AQP4 that was in parallel with GFAP changes. In spinal cord white matter, the overlap between AQP4 and GFAP is almost complete (Vitellaro-Zuccarello et al., 2005), suggesting that AQP4 is a binding partner of GFAP. Actin is a dynamic astrocytic cytoskeletal element (Wang and Hatton, 2007b). Astrocytic process elongation and branching are related to redistribution of both GFAP and actin filaments (Boran and Garcia, 2007). Thus, GFAP may link astrocytic membrane via molecular association with actin. Glu-Syn (Albrecht and Norenberg, 2006; Mong and Blutstein, 2006) and serine racemase (Oliet and Mothet, 2006) are both astrocytic enzymes involving glutamate metabolism or actions. Their expression levels and spatiotemporal distribution are important for astrocytic modulation of neuronal activity and may also be under intensive modulation of GFAP. To test these possibilities, coimmunoprecipitation of GFAP with AQP4, actin, Glu-Syn, and serine racemase was
$\frac{E}{\bar{J}}$

performed at different stages of suckling. The results from three replicates showed that suckling temporarily decreased GFAP associations with AQP4 (Fig. 7A) but increased GFAP association with actin (Fig. 7B), which were reversed after the MER. Suckling tended to reduce GFAP association with Glu-Syn after the MER (Fig. 7C). However, the association between GFAP and serine racemase (Fig. $7 D$ ) had no significant change (averaged changes, $<20 \%$ of non-suckling in three samples) despite decreases of serine racemase in confocal images during suckling and increases after the MER (data not shown). These results support the possibility that GFAP can influence OT neuronal activity by acutely changing molecular associations between GFAP and other functional proteins. It also suggests that serine racemase may not function via molecular interactions with GFAP.

\section{Discussion}

In the present study, we found that GFAP plasticity dynamically reflects OT neuronal activity via neurogenic neurochemical changes; this plasticity in turn modulates OT neuronal activity by changing GFAPassociated water transportation, morphology, and glutamate metabolism in astrocytes.

\section{GFAP plasticity during suckling}

GFAP is composed of head, rod (body), and tail domains and is posttranslationally modified by phosphorylation and proteolysis (Inagaki et al., 1990). GFAP can form different sized filaments via interconnection and/or bundling with other cytoskeletal components, e.g., vimentin (Mercier and Hatton, 2004). Depolymerized GFAP filaments can produce GFAP monomers, dimmers, and tetramers; dissembled and degraded GFAP monomers generate GFAP fragments, which may make GFAP invisible in light microscopic images at the initial period of suckling. GFAP usually appeared at $50 \mathrm{kDa}$ bands in Western blotting; however, degraded/dissembled GFAP may not be detected by antibody depending on whether GFAP antigens exist. Thus, the reversal of GFAP images after the MER should be related to simultaneous increases in reassembling of different domains and polymerization of GFAP filaments. It remains to be tested whether there is new protein synthesis. Meanwhile, there was also a trend toward increases in degrading or incomplete reassembling of GFAP because GFAP fragments also increased in abundance. This bidirectional result may reflect microdomain-specific GFAP dynamics, e.g., degraded/dissembled in the somata and assembled/polymerized in the processes (Fig. 3A).

Suckling increases somatodendritic release of OT, first in low levels and then to high levels (Neumann et al., 1993). In this study, a small increase of OT (10 pM) simulated the effect of suckling on GFAP at the initial stage; high levels of OT (1 nM) increased GFAP depolymerization and fragmentation. OT at high levels might activate proteases while increasing production of GFAP monomers but did not promote GFAP polymerization. Thus, the MER-elicited reexpansion of GFAP filaments must be attributable to some factors 
other than OT. Simulating the transient change in extracellular $\mathrm{K}^{+}$levels during synchronized bursts (Leng and Shibuki, 1987) revealed that high $\mathrm{K}^{+}$increased GFAP levels as seen in confocal microscopy and Western blotting. This finding is consistent with a previous report that cell swelling and increase in GFAP are evoked in isolated rat spinal cords by 50 mu K ${ }^{+}$(Syková et al., 1999). Thus, GFAP plasticity dynamically reflects patterned neuronal activity.

\section{GFAP plasticity and} astrocytic morphology Changes in GFAP stability primarily determine the morphology of astrocytes and gliosis (Weinstein et al., 1991; Pekny and Pekna, 2004; Boran and Garcia, 2007; Sizonenko et al., 2008). Using a suckling events-matched sampling method, we have revealed the dynamic process of GFAP plasticity during suckling. The plastic changes in GFAP filaments were accompanied by synergistic alterations in AQP4 expression during the initial stages of suckling and the MER in morphology and molecular interactions. Because AQP4 expression on astrocytic membranes is essential for water and ion transportation, a basis of cell swelling and shrinkage, the synergistic changes between GFAP and AQP4 support the proposal that GFAP expression reflects morphological changes in astrocytes at different stages of suckling. In fact, reduction of GFAP during suckling is temporally associated with the retraction of astrocytic processes, as reported previously (Wang and Hatton, 2007b). The question is whether GFAP reexpansion after the MER also indicates an expansion of astrocytic processes. Based on the morphology of immunostaining of filamentous actin between neighboring neurons in the $\mathrm{SON}$, our previous observation did not show a reexpansion of astrocytic processes after the MER (Wang and Hatton, 2007b). However, this finding did not exclude subtle or transient changes in the astrocytic processes on the MER attributable to the following factors. First, the resolution of present techniques may not be high enough to detect such a change. In immunostaining, the cellular apposition or retraction of astrocytic processes was defined as "intercellular space or the membrane filamentous actin thickness had to be $<1 \mu \mathrm{m}$ " (Wang and Hatton, 2007b), which is different from classical definition of glial retraction or cellular apposition ( $<200 \mathrm{~nm}$ ) (Hatton, 2004), based on electron microscopy. Thus, a change in the diameters of astrocytic processes $<1 \mu \mathrm{m}$ is not detectable with light microscopy. The subtle changes in astrocytic processes are likely because astrocytes have a well developed cytoskeleton, which makes astrocytes resistant to cell swelling in response to hyposmotic stimulation (Tomita et al., 2003). Second, the outlines of astrocytes are only partially represented by GFAP as Bauer Peptide-loading experiments show, and GFAP dynamics may represent only the "reactive" compartments of astrocytes, mainly at the processes. Thus, subtle reexpansion of GFAP-positive processes may be "buried" in a majority of GFAP-negative processes that may not reexpand in filamentous actin staining. Finally, GFAP plasticity and morphological changes in astrocytic processes may occur with different time courses. A transient astrocytic swelling may promote
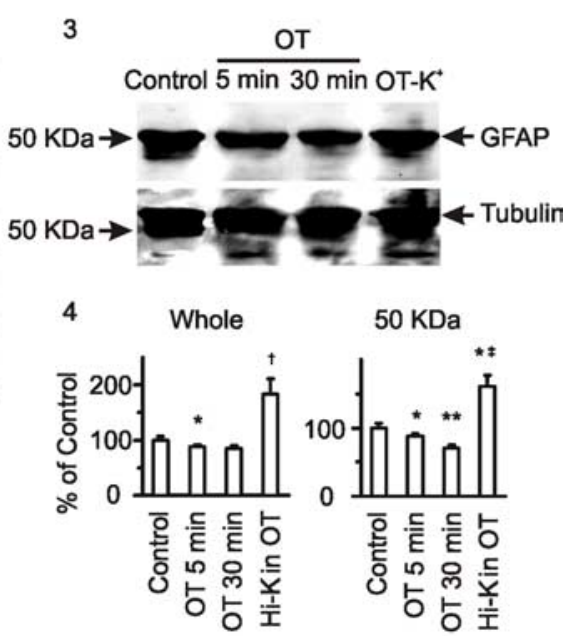

3
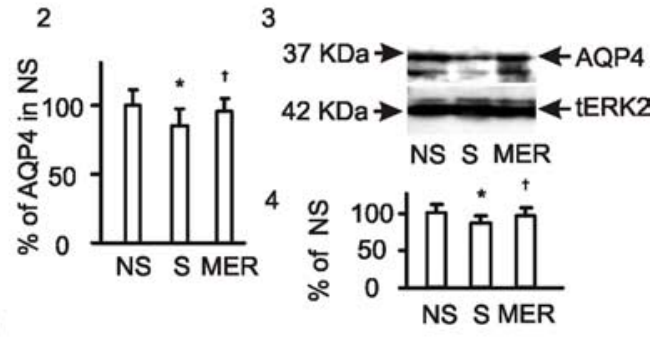

$20 \overline{\mu m}$

Figure 6. GFAP plasticity and homeostasis of $\mathrm{K}^{+}$/water in response to OT/suckling. $A$, Confocal microscopy of GFAP in the SON in slices. $\boldsymbol{A 1}$, Exemplary images of nuclei, GFAP, neurophysins (NPs), and their merges (from left to right) at control, 0 T ( $0.1 \mathrm{~nm}, 30$ ), and OT (20 min before) plus transient high $\mathrm{K}^{+}$stimulation (Hi-K; $\left.12 \mathrm{~mm}, 30 \mathrm{~s}\right)$. A2, Summary graphs $(n=6)$ showing relative intensities of GFAP. A3, A4, GFAP expression in Western blots and summary graphs $(n=4) .{ }^{\dagger} p<0.05$ and ${ }^{\ddagger} p<0.01$ compared with $0.1 \mathrm{nм}$ OT at $30 \mathrm{~min}$. $\boldsymbol{B}$, Effects of suckling on $\mathrm{AQP4}$ expression in confocal images $(\boldsymbol{B} \mathbf{n} ;=6)$ and in Western blots $(\boldsymbol{B} 3 ; n=3)$ and their summary graphs $(\boldsymbol{B} 2, \boldsymbol{B} 4)$, respectively. Other annotations refer to Figures 2 and 3.

GFAP reorganization, but the two were modulated differentially. In the brain, high $\mathrm{K}^{+}$after bursts can increase $\mathrm{K}^{+}$buffering in astrocytes (Kofuji and Newman, 2004) and AQP4 activity (Nagelhus et al., 2004), which leads to astrocytic swelling. Astrocytic swelling elicited by hypotonic stimulation can increase GFAP polymerization and AQP4 expression (our unpublished data), as high $\mathrm{K}^{+}$did, and rapidly facilitates regulatory volume decreases (Nabekura et al., 2003; Evanko et al., 2004) or volume transportation through the AQP4-containing astrocytic syncytium (Niermann et al., 2001). We assumed that extracellular $\mathrm{K}^{+}$elevation after bursts increases AQP4 activity first, which leads to astrocytic swelling and GFAP polymerization. The swelling is then quickly gone via regulatory volume decreases and/or volume transportation through astrocytic syncytium while GFAP filaments remain visible, resulting in the mismatch between astrocytic morphology and GFAP expression.

An unexpected result is that GFAP was negatively associated with actin in coimmunoprecipitation experiments. At the initial stage of suckling, despite a GFAP expression decrease, the molecular association between GFAP and actin increased, whereas, the partial recovery of GFAP levels reduced such an association. It seems that GFAP and astrocytic expansion need to remove a potential barrier effect of filamentous actin, although association between GFAP and actin is needed to retract membranes of astrocytic processes. In agreement are this and the finding that hypotonic stimulation and glial swelling are accompanied by actin depolymerization (Lascola and Kraig, 1996; Morán et al., 1996). It is important to further clarify the physiological significance of the transient changes in the association between GFAP, AQP4, and actin during suckling. 

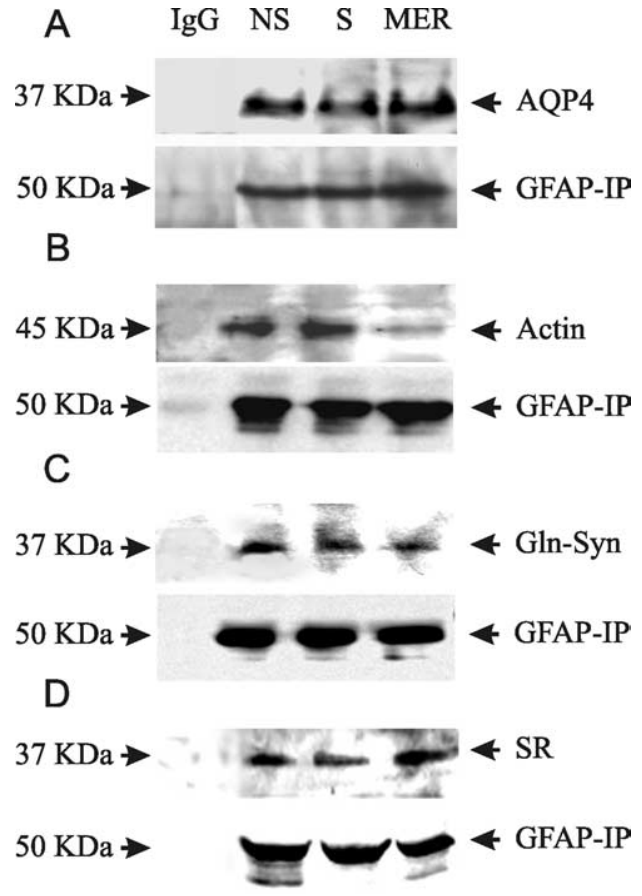

Figure 7. Molecular association between GFAP and other astrocytic structural and functional molecules at different stages of suckling. $A-D$, Immunoprecipitation of GFAP (mouse antibody, bottom) and detection of AQP4 $(\boldsymbol{A})$, actin $(\boldsymbol{B})$, Glu-Syn $(\boldsymbol{C})$, and serine racemase (SR; $\boldsymbol{D})$ with rabbit antibodies in Western blotting, respectively. Mouse lgG was used as negative control and total lysates or purified proteins as positive controls. Other annotations refer to Figure 2. IP, Immunoprecipitation.

\section{GFAP plasticity and glutamate metabolism/actions}

Glutamate plays a central role in astrocytic neuronal interactions (Swanson et al., 2004). Astrocytes modulate neurotransmission via release of glutamate (Vesce et al., 2007) and D-serine (Martineau et al., 2006) in soluble $N$-ethylmaleimide-sensitive factor attachment protein receptor- and $\mathrm{Ca}^{2+}$-dependent manners (Takahashi et al., 2007). In the present study, TeTx blocked most of the spontaneous EPSCs and left only a few miniature EPSCs that were insensitive to OT. EPSCs, particularly those occurring in clustered patterns, were sensitive to L-AAA. Because the clustered EPSCs are likely a major OT neuronal burst trigger (Wang and Hatton, 2004, 2007a), astrocytic glutamate metabolism and actions should play an important role in the regulation of OT neuronal activity. Interestingly, the duration and frequency but not amplitude of EPSC clustering play key roles in burst generation shown by L-AAA actions.

Molecular interactions between GFAP and Glu-Syn and serine racemase participate in glutamate metabolism/actions. The decreased association between GFAP and Glu-Syn during suckling suggests a reduced molecular interaction between these two molecules. In general, localization of GFAP is relatively stable; the association of GFAP with Glu-Syn may restrain the conversion of glutamate to glutamine around GFAP filaments. The retraction of GFAP during suckling may reduce the conversion and increase extracellular ambient glutamate; the reduction of molecular association during reexpansion of GFAP at the MER may allow this enzyme to function beyond the limits of GFAP and quick removal of extracellular glutamate. In contrast, serine racemase has no clear trend of molecular association with GFAP during suckling. In immunostaining, the expression of this enzyme was seen also in magnocellular compartments and putative microglia in the SON (our unpublished data). Thus, the involvement of D-serine in the MER may not be limited to the astrocytes. Moreover, L-AAA inhibited the MER but increased the amplitude of clustering EPSCs, suggesting that D-serine promotion of NMDA receptor activation may not be critical for burst generation. The functions of GFAP are associated with glutamate transporters, as shown by L-AAA actions and in other systems (Sullivan et al., 2007). Although the roles of different types of glutamate transporters remains to be explored in the SON during suckling, it is likely that suckling may decrease the expression/or function of these transporters, whereas the occurrence of MER partially restore them.

\section{Implications of studying astrocytic plasticity}

Increases in OT secretion reduce GFAP expression and cause retraction of astrocytic processes, exerting the initial excitatory effects of suckling. After synchronized bursts before the MER, high level of OT and transient high $\mathrm{K}^{+}$increase GFAP expression, which helps to maintain the homeostasis of the extracellular neurochemical environment but leads to transient postburst inhibition. Similar mechanisms may be applicable for glial-neuronal interactions in other neural systems, although the neurotransmitters may vary.

\section{References}

Albrecht J, Norenberg MD (2006) Glutamine: a Trojan horse in ammonia neurotoxicity. Hepatology 44:788-794.

Amiry-Moghaddam M, Williamson A, Palomba M, Eid T, de Lanerolle NC, Nagelhus EA, Adams ME, Froehner SC, Agre P, Ottersen OP (2003) Delayed $\mathrm{K}^{+}$clearance associated with aquaporin- 4 mislocalization: phenotypic defects in brains of alpha-syntrophin-null mice. Proc Natl Acad Sci U S A 100:13615-13620.

Anderová M, Kubinová S, Mazel T, Chvátal A, Eliasson C, Pekny M, Syková E (2001) Effect of elevated $\mathrm{K}(+)$, hypotonic stress, and cortical spreading depression on astrocyte swelling in GFAP-deficient mice. Glia 35:189-203.

Armstrong WE, Rubrum A, Teruyama R, Bond CT, Adelman JP (2005) Immunocytochemical localization of small-conductance, calciumdependent potassium channels in astrocytes of the rat supraoptic nucleus. J Comp Neurol 491:175-185.

Badaut J, Nehlig A, Verbavatz J, Stoeckel M, Freund-Mercier MJ, Lasbennes F (2000) Hypervascularization in the magnocellular nuclei of the rat hypothalamus: relationship with the distribution of aquaporin- 4 and markers of energy metabolism. J Neuroendocrinol 12:960-969.

Baudoux S, Parker D (2008) Glial-toxin-mediated disruption of spinal cord locomotor network function and its modulation by 5 -HT. Neuroscience 153:1332-1343

Belin V, Moos F (1986) Paired recordings from supraoptic and paraventricular oxytocin cells in suckled rats: recruitment and synchronization. J Physiol 377:369-390.

Binder DK, Yao X, Zador Z, Sick TJ, Verkman AS, Manley GT (2006) Increased seizure duration and slowed potassium kinetics in mice lacking aquaporin-4 water channels. Glia 53:631-636.

Borán MS, García A (2007) The cyclic GMP-protein kinase G pathway regulates cytoskeleton dynamics and motility in astrocytes. J Neurochem 102:216-230.

Catheline G, Touquet B, Lombard MC, Poulain DA, Theodosis DT (2006) A study of the role of neuro-glial remodeling in the oxytocin system at lactation. Neuroscience 137:309-316.

Crowley WR, Armstrong WE (1992) Neurochemical regulation of oxytocin secretion in lactation. Endocr Rev 13:33-65.

de Kock CP, Wierda KD, Bosman LW, Min R, Koksma JJ, Mansvelder HD, Verhage M, Brussaard AB (2003) Somatodendritic secretion in oxytocin neurons is upregulated during the female reproductive cycle. J Neurosci 23:2726-2734.

Dieck ST, Heuer H, Ehrchen J, Otto C, Bauer K (1999) The peptide transporter PepT2 is expressed in rat brain and mediates the accumulation of the fluorescent dipeptide derivative beta-Ala-Lys-Nepsilon-AMCA in astrocytes. Glia 25:10-20.

Ding M, Eliasson C, Betsholtz C, Hamberger A, Pekny M (1998) Altered taurine release following hypotonic stress in astrocytes from mice deficient for GFAP and vimentin. Brain Res Mol Brain Res 62:77-81.

Espallergues J, Solovieva O, Técher V, Bauer K, Alonso G, Vincent A, Hussy N (2007) Synergistic activation of astrocytes by ATP and norepinephrine in the rat supraoptic nucleus. Neuroscience 148:712-723. 
Evanko DS, Zhang Q, Zorec R, Haydon PG (2004) Defining pathways of loss and secretion of chemical messengers from astrocytes. Glia 47:233-240.

Gimpl G, Fahrenholz F (2001) The oxytocin receptor system: structure, function, and regulation. Physiol Rev 81:629-683.

Gommerat I, Gola M (1996) Glial potassium channels activated by neuronal firing or intracellular cyclic AMP in Helix. J Physiol 495:649-664.

Halpern JL, Habig WH, Trenchard H, Russell JT (1990) Effect of tetanus toxin on oxytocin and vasopressin release from nerve endings of the neurohypophysis. J Neurochem 55:2072-2078.

Hatton GI (2004) Morphological plasticity of astroglial/neuronal interactions: functional implications. In: Glial neuronal signaling (Hatton GI, Parpura V, eds), pp 99-124. Boston: Kluwer Academic.

Hatton GI, Yang QZ, Cobbett P (1987) Dye coupling among immunocytochemically identified neurons in the supraoptic nucleus: increased incidence in lactating rats. Neuroscience 21:923-930.

Hughes EG, Maguire JL, McMinn MT, Scholz RE, Sutherland ML (2004) Loss of glial fibrillary acidic protein results in decreased glutamate transport and inhibition of PKA-induced EAAT2 cell surface trafficking. Brain Res Mol Brain Res 124:114-123.

Inagaki M, Gonda Y, Nishizawa K, Kitamura S, Sato C, Ando S, Tanabe K, Kikuchi K, Tsuiki S, Nishi Y (1990) Phosphorylation sites linked to glial filament disassembly in vitro locate in a non-a-helical head domain. J Biol Chem 265:4722-4729.

Kofuji P, Newman EA (2004) Potassium buffering in the central nervous system. Neuroscience 129:1045-1056.

Lascola CD, Kraig RP (1996) Whole-cell chloride currents in rat astrocytes accompany changes in cell morphology. J Neurosci 16:2532-2545.

Leng G, Shibuki K (1987) Extracellular potassium changes in the rat neurohypophysis during activation of the magnocellular neurosecretory system. J Physiol 392:97-111.

Li L, Lundkvist A, Andersson D, Wilhelmsson U, Nagai N, Pardo AC, Nodin C, Ståhlberg A, Aprico K, Larsson K, Yabe T, Moons L, Fotheringham A, Davies I, Carmeliet P, Schwartz JP, Pekna M, Kubista M, Blomstrand F, Maragakis N, Nilsson M, Pekny M (2008) Protective role of reactive astrocytes in brain ischemia. J Cereb Blood Flow Metab 28:468-481.

Martineau M, Baux G, Mothet JP (2006) D-serine signalling in the brain: friend and foe. Trends Neurosci 29:481-491.

McCall MA, Gregg RG, Behringer RR, Brenner M, Delaney CL, Galbreath EJ, Zhang CL, Pearce RA, Chiu SY, Messing A (1996) Targeted deletion in astrocyte intermediate filament (Gfap) alters neuronal physiology. Proc Natl Acad Sci U S A 93:6361-6366.

Mercier F, Hatton GI (2004) Meninges and perivasculature as mediators of CNS plasticity. In: Advances in molecular and cellular biology, Ed 1 (Hertz L, ed), pp 215-253. San Diego: Elsevier.

Mong JA, Blutstein T (2006) Estradiol modulation of astrocytic form and function: implications for hormonal control of synaptic communication. Neuroscience 138:967-975.

Morán J, Sabanero M, Meza I, Pasantes-Morales H (1996) Changes of actin cytoskeleton during swelling and regulatory volume decrease in cultured astrocytes. Am J Physiol 271:C1901-C1907.

Nabekura T, Morishima S, Cover TL, Mori S, Kannan H, Komune S, Okada Y (2003) Recovery from lactacidosis-induced glial cell swelling with the aid of exogenous anion channels. Glia 41:247-259.

Nagelhus EA, Mathiisen TM, Ottersen OP (2004) Aquaporin-4 in the central nervous system: cellular and subcellular distribution and coexpression with KIR4.1. Neuroscience 129:905-913.

Neumann I, Russell JA, Landgraf R (1993) Oxytocin and vasopressin release within the supraoptic and paraventricular nuclei of pregnant, parturient and lactating rats: a microdialysis study. Neuroscience 53:65-75.

Niermann H, Amiry-Moghaddam M, Holthoff K, Witte OW, Ottersen OP (2001) A novel role of vasopressin in the brain: modulation of activitydependent water flux in the neocortex. J Neurosci 21:3045-3051.

Nishida H, Okabe S (2007) Visualization of synapse-glia dynamics (in Japanese). Brain Nerve 59:755-761.

Okere CO, Wang YF, Higuchi T, Negoro H, Okutani F, Takahashi S, Murata $\mathrm{T}$ (1996) The effect of systemic and central nitric oxide administration on milk availability in lactating rats. Neuroreport 8:243-247.

Oliet SH, Mothet JP (2006) Molecular determinants of D-serine-mediated gliotransmission: from release to function. Glia 54:726-737.

Pannicke T, Stabel J, Heinemann U, Reichelt W (1994) alpha-Aminoadipic acid blocks the $\mathrm{Na}^{+}$-dependent glutamate transport into acutely isolated Muller glial cells from guinea pig retina. Pflugers Arch 429:140-142.
Paxinos G, Watson C (1998) The rat brain in stereotaxic coordinates, Ed 4. New York: Academic.

Pekny M, Pekna M (2004) Astrocyte intermediate filaments in CNS pathologies and regeneration. J Pathol 204:428-437.

Pekny M, Wilhelmsson U, Bogestål YR, Pekna M (2007) The role of astrocytes and complement system in neural plasticity. Int Rev Neurobiol 82:95-111.

Pierre K, Bonhomme R, Dupouy B, Poulain DA, Theodosis DT (2001) The polysialylated neural cell adhesion molecule reaches cell surfaces of hypothalamic neurons and astrocytes via the constitutive pathway. Neuroscience 103:133-142.

Rosso L, Peteri-Brunbäck B, Vouret-Craviari V, Deroanne C, Van Obberghen-Schilling E, Mienville JM (2002) Vasopressin and oxytocin reverse adenosine-induced pituicyte stellation via calcium-dependent activation of Cdc42. Eur J Neurosci 16:2324-2332.

Rosso L, Peteri-Brunbäck B, Mienville JM (2004) Putative physiological significance of vasopressin V1a receptor activation in rat pituicytes. J Neuroendocrinol 16:313-318.

Shibuki K, Gomi H, Chen L, Bao S, Kim JJ, Wakatsuki H, Fujisaki T, Fujimoto K, Katoh A, Ikeda T, Chen C, Thompson RF, Itohara S (1996) Deficient cerebellar long-term depression, impaired eye blink conditioning, and normal motor coordination in GFAP mutant mice. Neuron 16:587-599.

Simard M, Nedergaard M (2004) The neurobiology of glia in the context of water and ion homeostasis. Neuroscience 129:877-896.

Sizonenko SV, Camm EJ, Dayer A, Kiss JZ (2008) Glial responses to neonatal hypoxic-ischemic injury in the rat cerebral cortex. Int J Dev Neurosci 26:37-45.

Sullivan SM, Lee A, Björkman ST, Miller SM, Sullivan RK, Poronnik P, Colditz PB, Pow DV (2007) Cytoskeletal anchoring of GLAST determines susceptibility to brain damage: an identified role for GFAP. J Biol Chem 282:29414-29423.

Swanson RA, Ying W, Kauppinen TM (2004) Astrocyte influences on ischemic neuronal death. Curr Mol Med 4:193-205.

Syková E, Vargová L, Prokopová S, Simonová Z (1999) Glial swelling and astrogliosis produce diffusion barriers in the rat spinal cord. Glia 25:56-70.

Takahashi M, Itakura M, Yamamori S (2007) Exocytotic release of signaling molecules from astrocytes (in Japanese). Brain Nerve 59:697-706.

Tekkok SB, Ransom BR (2004) The glial-neuronal interactions and signaling: an introduction. In: Glial neuronal signaling (Hatton GI, Parpura V, eds), pp 1-20. Boston: Kluwer Academic.

Theodosis DT, Schachner M, Neumann ID (2004) Oxytocin neuron activation in NCAM-deficient mice: anatomical and functional consequences. Eur J Neurosci 20:3270-3280.

Tomita M, Tanahashi N, Takeda H, Takao M, Tomita Y, Amano T, Fukuuchi Y (2003) Astroglial swelling in the neuronal depolarization ensemble. Acta Neurochir Suppl 86:219-222.

Vesce S, Rossi D, Brambilla L, Volterra A (2007) Glutamate release from astrocytes in physiological conditions and in neurodegenerative disorders characterized by neuroinflammation. Int Rev Neurobiol 82:57-71.

Vitellaro-Zuccarello L, Mazzetti S, Bosisio P, Monti C, De Biasi S (2005) Distribution of Aquaporin 4 in rodent spinal cord: relationship with astrocyte markers and chondroitin sulfate proteoglycans. Glia 51:148-159.

Wakerley JB, Clarke G, Summerlee AJ (1994) Milk ejection and its control. In: The physiology of reproduction (Knobil E, Neill JD, eds), pp 11311177. New York: Raven.

Walz W, Wuttke WA (1999) Independent mechanisms of potassium clearance by astrocytes in gliotic tissue. J Neurosci Res 56:595-603.

Wang YF, Hatton GI (2004) Milk ejection burst-like electrical activity evoked in supraoptic oxytocin neurons in slices from lactating rats. J Neurophysiol 91:2312-2321.

Wang YF, Hatton GI (2006) Mechanisms underlying oxytocin-induced excitation of supraoptic neurons: prostaglandin mediation of actin polymerization. J Neurophysiol 95:3933-3947.

Wang YF, Hatton GI (2007a) Dominant role of $\beta \gamma$ subunits of G-proteins in oxytocin-evoked burst firing. J Neurosci 27:1902-1912.

Wang YF, Hatton GI (2007b) Interaction of extracellular signal-regulated protein kinase $1 / 2$ with actin cytoskeleton in supraoptic oxytocin neurons and astrocytes: role in burst firing. J Neurosci 27:13822-13834.

Weinstein DE, Shelanski ML, Liem RKH (1991) Suppression by antisense mRNA demonstrates a requirement for the glial fibrillary acidic protein in the formation of stable astrocytic processes in response to neurons. J Cell Biol 112:1205-1213. 